Results All participants $(100 \% ; n=23)$ indicated willingness to provide samples for STI screening during a future study visit. Participant preference was for self-collection of urine samples $(82.6 \% ; \mathrm{n}=19)$, urethral swabs $(81.8 \% ; \mathrm{n}=18)$, and anorectal swabs $(77.3 \% ; \mathrm{n}=17)$. A lower preference for self-collection of oropharyngeal swabs $(47.8 \% ; n=11)$ was observed. Most respondents $(78.3 \% ; \mathrm{n}=18)$ indicated that they would not prefer sampling to be collected by a health professional, mainly due to 'more privacy' $(72.2 \% ; n=13)$. All participants $(n=20 ; 3$ missing) indicated that they would feel comfortable to provide a self-collected sample based on instructional diagrams shown.

Conclusion This study suggested acceptability among transgender women of introducing self-collected sampling for etiological diagnosis of STIs from potential infection sites. Novel gender-neutral instructional diagrams received positive responses of understanding to enable self-collected samples, with further development and testing warranted. Uptake and usability will be explored in TransOdara, a cross-sectional STI prevalence study of transgender women in Brazil.

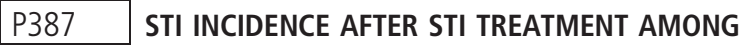 WOMEN AT RISK FOR HIV EXPOSURE INITIATING SAFER CONCEPTION CARE IN SOUTHWESTERN UGANDA}

${ }^{1} \mathrm{P}$ Chitneni ${ }^{*},{ }^{2} \mathrm{M}$ Bwana, ${ }^{3} \mathrm{M}$ Owembabazi, ${ }^{3} \mathrm{~K}$ O'Neil, ${ }^{2} \mathrm{P}$ Kalyebara, ${ }^{2} \mathrm{~W}$ Muyindike, ${ }^{2} \mathrm{~A}$ Byamukama, ${ }^{2} \mathrm{Y}$ Mbalibulha, ${ }^{4} \mathrm{~K}$ Wirth, ${ }^{5} \mathrm{D}$ Bangsberg, ${ }^{6} \mathrm{~J}$ Marrazzo, ${ }^{7} \mathrm{~J}$ Haberer, ${ }^{8} \mathrm{~A}$ Kaida, ${ }^{6} \mathrm{~L}$ Matthews. 'Brigham and Women's Hospital, Boston, USA; ${ }^{2}$ Mbarara Regional Referral Hospital and Mbarara University of Science and Technology, Mbarara, Uganda; ${ }^{3}$ Global Health Collaborative, Mbarara, Uganda; ${ }^{4}$ Botswana Harvard AIDS Institute Partnership, Gaborone, Botswana; ${ }^{5}$ Oregon Health Sciences University and Portland State University School of Public Health, Portland, USA; ${ }^{6}$ University of Alabama at Birmingham, Birmingham, USA; ${ }^{7}$ Massachusetts General Hospital, Boston, USA; ${ }^{8}$ Simon Fraser University, Burnaby, Canada

\subsection{6/sextrans-2021-sti.422}

Background Sexually transmitted infection (STI) recurrence contributes to the high global STI burden. We introduced STI screening and facilitated partner notification $(\mathrm{PN})$ and treatment among women participating in a safer conception study in southwestern Uganda to understand impacts on STI incidence.

Methods A parent study enrolled women planning for pregnancy with a man with HIV or of unknown serostatus to assess pre-exposure prophylaxis use for safer conception. STI screening began after study-start, and all eligible women completed screening for chlamydia, gonorrhea, and trichomoniasis via GeneXpert nucleic acid amplification testing and syphilis via immunochromatographic testing and rapid plasma reagin. Multivariable Poisson regression was used to determine incident STI correlates.

Results Of 134 women in the parent study, 94 underwent enrollment STI screening, of whom 23 were positive. Median age was 31 (IQR 28-35) years. All participants with STIs received counseling and treatment; 21/23 participants accepted PN cards and 18/23 accepted patient-delivered partner medications. By the six-month study-visit, 81 participants repeated STI testing ( $\mathrm{N}=66$ at that visit, $\mathrm{N}=15$ at incident pregnancy visit; whichever came first); 13 participants were lost to follow-up. Of those with enrollment STIs, 19/23 returned for follow-up at six months; 18 reported delivering PN cards and discussing STIs with partner(s) and 14 reported medication delivery to partner(s). Incident STIs occurred in $17 / 81$ participants with 42.57 person-years of follow-up (incidence rate $40 / 100$ person-years). STI incidence was associated with enrollment STI (incidence rate ratio [IRR] 3.39, 95\% confidence interval [CI] 1.22-9.43) and alcohol consumption (IRR 3.18, 95\% CI 1.15-8.85).

Conclusions We demonstrate a high STI prevalence and incidence among women planning for pregnancy in Uganda despite partner treatment promotion efforts. These infections are likely driven in part by re-infection from untreated partners. Novel STI PN interventions are needed to decrease the STI burden, especially among women planning for and with pregnancy.

\section{P388 MYCOPLASMA GENITALIUM-REACTIVE ANTIBODIES IN SERUM AND URETHRAL SPECIMENS OF PERSISTENTLY INFECTED MEN}

${ }^{1} \mathrm{C}$ Kim*, ${ }^{2,3} \mathrm{~L}$ Manheart, ${ }^{2} \mathrm{C}$ Gillespie, ${ }^{2} \mathrm{C}$ Khosropour, ${ }^{4} \mathrm{M}$ Lowens, ${ }^{5,6} \mathrm{P}$ Totten, ${ }^{1} \mathrm{G}$ Wood. ${ }^{\prime}$ Department of Medicine, Division of Allergy and Infectious Diseases, University of Washington, Seattle, USA; ${ }^{2}$ Department of Epidemiology, University of Washington, Seattle, USA; ${ }^{3}$ Department of Global Health, University of Washington, Seattle, USA; ${ }^{4}$ Public Health -Seattle and King County, Seattle, USA; ${ }^{5}$ Department of Medicine, Division of Allergy and Infectious Diseases, University of Washington, Seattle, USA; ${ }^{6}$ Department of Global Health, Pathobiology Interdisciplinary Program, University of Washington, Seattle, USA

\subsection{6/sextrans-2021-sti.423}

Background Mycoplasma genitalium (MG) is a sexually transmitted pathogen associated with acute and chronic genital tract infection in men and women. Although MG-reactive antibodies have been detected in cervicovaginal specimens of infected women, antibodies at the site of infection in men have not been characterized. We compared MG-reactive antibodies in serum and urethral swabs of MG positive and negative men with urethritis to better understand the immunopathogenesis of MG.

Methods Paired urethral swab and serum specimens were collected from men enrolled in a double-blinded study comparing azithromycin and doxycycline for treatment of non-gonococcal urethritis conducted from 2007-2011. Among 22 MG-positive men, antibody reactivity to MG was determined by immunoblot for specimens collected at enrollment and the last MGpositive visit (15-86 days later). Paired specimens from 13 MG-negative men served as controls.

Results In serum specimens, MG-reactive antibodies were detected in all 22 MG-positive men. In 10 men, increasing reactivity to the $\mathrm{MgpB}$ and $\mathrm{MgpC}$ immunodominant adherence proteins occurred over time. In comparison, sera from MG-negative men reacted poorly (7/13) or not at all (6/13) to these proteins. In urethral specimens, MG-specific antibody was detected in 22/22 MG-positive men demonstrating these antibodies are present at the site of infection. Surprisingly, antibody reactivity in urethral specimens decreased over time in $12 / 19$ patients despite the persistence of antibodies in serum and $\mathrm{MG}$ in the urethra. Among MG-negative men, 8/ 13 had detectable $\mathrm{MgpB} / \mathrm{MgpC}$ reactive antibody in urethral specimens but reactivity was very low.

Conclusion Serum antibodies to MG correlated well with infection status, but local antibodies did not. Despite the persistence of $\mathrm{MG}$ in the urethra, antibody reactivity at the site of infection diminished over time. Whether this reduced reactivity reflects reduced bacterial load over time, or whether diminution of local antibodies contributes to persistent infection should be examined in future studies. 